\title{
Microscopy Tools and Techniques Used in the Study of Infectious Disease Agents
}

\author{
Elizabeth R. Fischer ${ }^{1}$, Bryan T. Hansen ${ }^{1}$, Cindi L. Schwartz ${ }^{1}$, Vinod Nair ${ }^{1}$ \\ ${ }^{1 .}$ NIH/NIAID/Rocky Mountain Labs, Hamilton, USA.
}

Microscopes allow visualization of an unseen world. The discovery of microbes in the $17^{\text {th }}$ century as described by Robert Hooke and Antonie van Leeuwenhoek birthed the field of microbiology. As the germ theory evolved through the centuries, microscopes were critical in the identification and classification of infectious disease agents. With the invention of the electron microscope by Ernst Ruska in the $20^{\text {th }}$ century, the ability to resolve structures allowed visualization of even smaller particles including viruses and proteins. Today developments in both preparative microscopy tools and techniques combined with modern biochemical and molecular methods have significantly improved our ability to relate structure to function to nearly atomic level greatly facilitating our understanding human pathogens.

Conventional room temperature (RT) transmission (TEM) and scanning (SEM) electron microscope studies still provide useful morphological information. For instance, the trafficking of lipid droplets by signals from intracellular pathogens such as chlamydia can be discerned through conventional techniques. (Fig.1)

Labeling antigens for visualization by electron microscopy presents many obstacles due to the necessary compromise between antigen stability and ultra-structural preservation. Immune-labeling prior to embedding for accessible antigens allows for mild pre-fixation conditions, followed by immune reactions and the subsequent use of stronger fixatives to optimize ultrastructure. An additional advantage to immune-labeling prior to embedding is that reactions can be confirmed by light or fluorescent microscopy prior to the time-consuming processes of embedding and sectioning. Transient events can be correlated using correlative light and electron microscopy (CLEM) techniques. Fig. 2 illustrates CLEM strategy.

Cryo-preservation has long been regarded as the preferred technique for optimal preservation of structures in their native state, retaining components that would be lost or altered during conventional chemical fixation. Current developments and applications utilized in the study of infectious disease agents include high pressure freezing, plunge freezing, freeze fracture, and the ability to view specimens under room temperature and cryo-conditions by both SEM and high-resolution TEM. (Fig. 3).

Technology developments including phase-plates and direct detectors have enabled superior 2dimensional imaging for single particle analysis and improved cryo-electron tomography providing maximum ultrastructural 3-dimensional resolution. These improvements allow visualization of a variety of specimens including macromolecular and enzyme complexes, neutralizing antibody docking sites on viruses, 3-dimensional structures of intact viruses, and intracellular relationships between viruses and bacteria as they enter, replicate, and exit cells, clearly of relevance in the study of human pathogens. (Fig. 4) [3]

References:

[1] Cocchiaro JL et al, PNAS 105(27) (2008), p. 9379. 
[2] Chlanda P et al, Nat Microbiol. 1(6) (2016), p. 16050.

[3] This work was supported by the NIAID intramural research program.
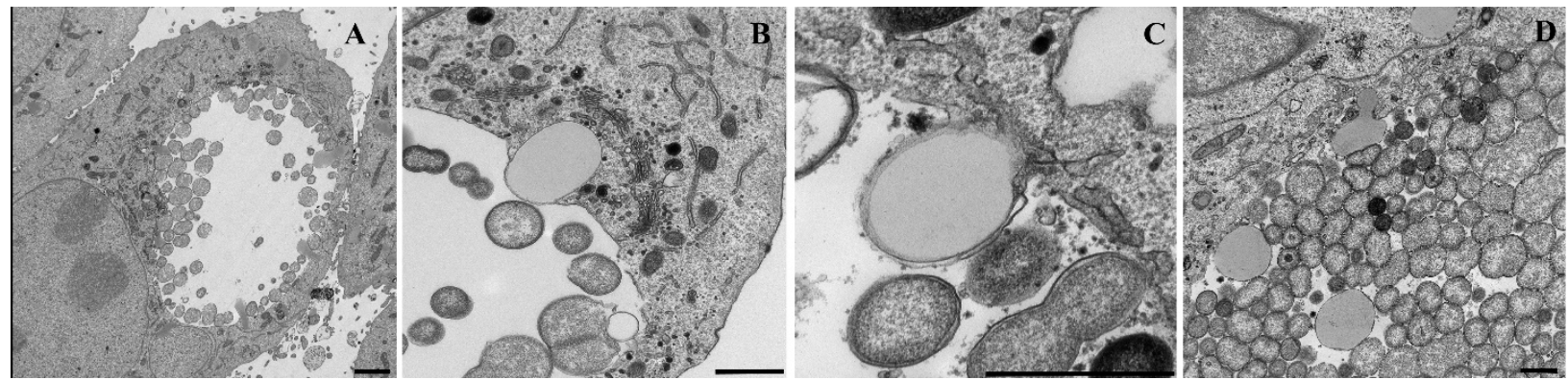

Figure 1. Lipid droplets show random distribution throughout the cytoplasm of HeLa cells infected with chlamydiae (A). As the inclusion vacuole matures, lipids are often seen in apparent transport across the inclusion membrane (B-D). Although EM cannot reveal the direction of travel, the lipid droplets appear to translocate across the inclusion membrane from the host cytosol. Addition of malachite green in chemical processing for EM may provide a useful technique for studying lipid droplet association with intracellular pathogens. Scale bars: $A=2 \mu \mathrm{m}, \mathrm{B}-\mathrm{D}=1 \mu \mathrm{m}$. [Ref.1]
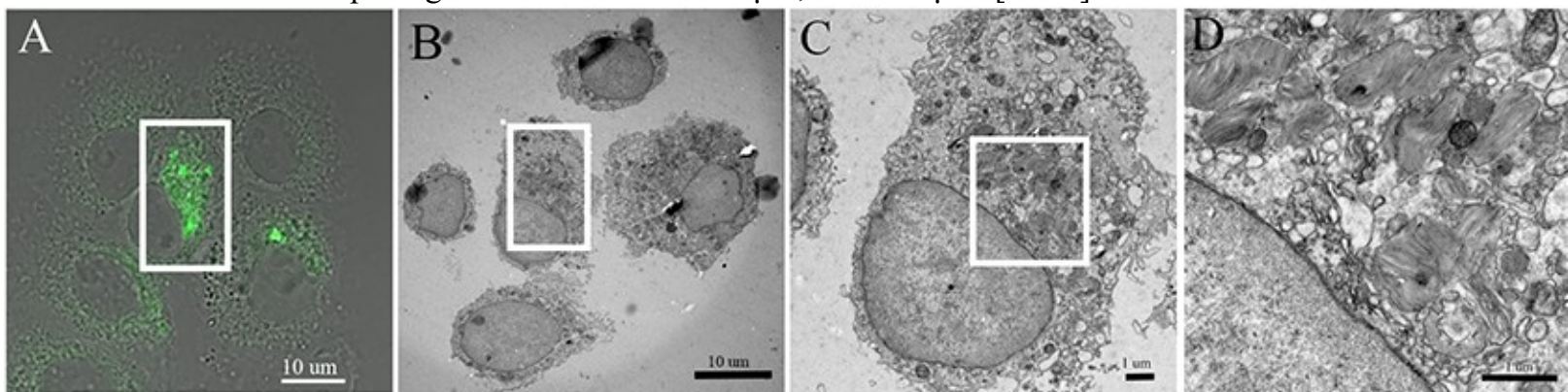

Figure 2. RMLs' Katy Bosio lab produced a synthetic form of a lipid that could recapitulate the anti-inflammatory effects observed with a native Francisella tularensis lipid. As part of these studies it was important to determine the intracellular localization of the synthetic lipid and determine how long the lipid was retained within the host cell. Using a correlative light and electron microscopy techniques (A-C) our group identified lipid laden primary human dendritic cells and determined the localization of the synthetic lipid within each cell (D).
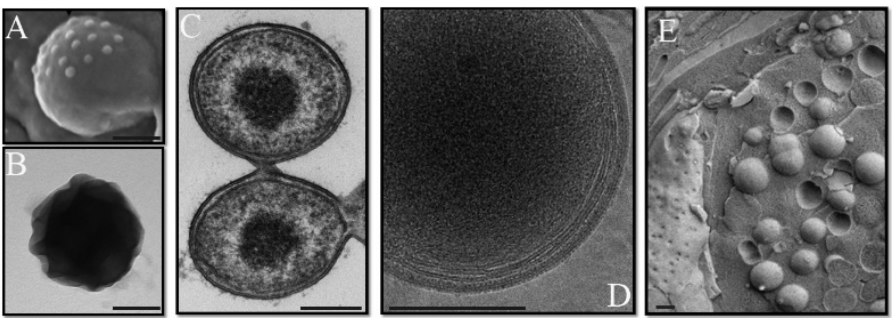

Figure 3. SEM (A), TEM negative stain (B) and thin sections (C) can provide useful information but cryo-plunge freezing (D) or high-pressure frozen freeze-fracture cryo-SEM (E) may be critical for high resolution studies of organisms such as chlamydiae shown here. Scale bars $=100 \mathrm{~nm}$
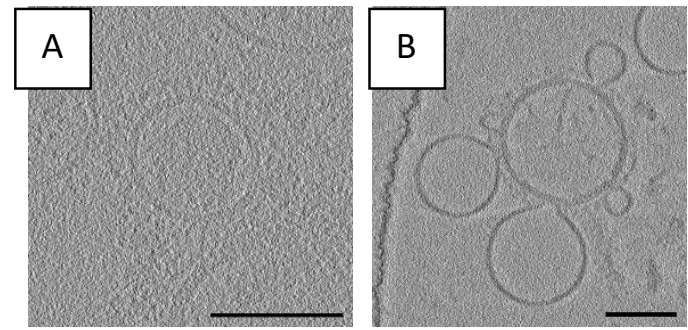

Figure 4. Contrast comparison between $-5 \mu \mathrm{m}$, no phase plate (A) and $-1 \mu \mathrm{m}$, with FEI Volta phase plate on $300 \mathrm{kV}$ Titan Krios. Scale bars $=100 \mathrm{~nm}$. [Ref. 2] 